\title{
Nanoengineered nonuniform strain in graphene using nanopillars
}

\author{
M. Neek-Amal, ${ }^{1,2}$ L. Covaci, ${ }^{2}$ and F. M. Peeters ${ }^{2}$ \\ ${ }^{1}$ Shahid Rajaee Teacher Training University, Lavizan, Tehran 16785-136, Iran \\ ${ }^{2}$ Departement Fysica, Universiteit Antwerpen, Groenenborgerlaan 171, B-2020 Antwerpen, Belgium
}

(Received 23 April 2012; published 12 July 2012)

\begin{abstract}
Recent experiments showed that nonuniform strain can be produced by depositing graphene over pillars. We employed atomistic calculations to study the nonuniform strain and the induced pseudomagnetic field in graphene on top of nanopillars. By decreasing the distance between the nanopillars a complex distribution for the pseudomagnetic field can be generated. Furthermore, we performed tight-binding calculations of the local density of states (LDOS) by using the relaxed graphene configuration obtained from atomistic calculations. We find that the quasiparticle LDOS are strongly modified near the pillars, both at low energies showing sublattice polarization and at high energies showing shifts of the van Hove singularity. Our study shows that changing the specific pattern of the nanopillars allows us to create a desired shape of the pseudomagnetic field profile while the LDOS maps provide an input for experimental verification by scanning tunneling microscopy.
\end{abstract}

DOI: 10.1103/PhysRevB.86.041405

PACS number(s): 61.48.Gh, 71.10.Pm

Graphene is a newly discovered atomic thin twodimensional honeycomb lattice consisting of carbon atoms. ${ }^{1}$ It is a zero gap semimetal with a conical band structure where the conduction and valence bands touch each other at the Dirac point. ${ }^{2}$ Nanoengineered nonuniform strain distribution in graphene is a promising road to generate a band gap and a large pseudomagnetic field. Scanning tunneling microscopy (STM) measurements have shown strain-induced Landau levels ${ }^{3}$ which correspond to a huge pseudomagnetic field on the order of $300 \mathrm{~T}$. Shear strain is essential and neither uniaxial nor isotropic strain produces a strong uniform pseudomagnetic field. ${ }^{4}$

Graphene's high responses to external forces result in mechanical deformations. Over the past few years there have been several efforts to control graphene's electronic properties by strain. ${ }^{5-7}$ Elastic deformations create a pseudomagnetic field which acts on graphene's massless charge carriers. ${ }^{2,4,8,9}$ The resulting variation of the hopping energies can be viewed as an induced pseudomagnetic field which enters in the Dirac equation. Engineering of the right topology of the induced pseudomagnetic field provides symmetrical magnetic confinement which confines electrons in specific regions in space. ${ }^{10}$

Recently, it was predicted that nonuniform strain may lead to a considerable energy gap and a large gauge field that effectively acts as a uniform magnetic field. ${ }^{11}$ Recently, Tomori et al. used pillars made of a dielectric material (electron beam resist) which were placed on top of a substrate that was then overlayed with graphene to generate nonuniform strain on a microscale. ${ }^{12}$ The graphene sections, which are located between the pillars, are attached to the substrate, and the size and separation of the pillars control the strength and distribution of the strain. The length scale in the experiment was micrometers and $\mathrm{SiO}_{2}$ was used as the substrate.

Here we study nonuniform strain at the atomistic scale where the continuum approach is no longer applicable. We also study the local density of state (LDOS) maps using the relaxed graphene configuration as the input for tight-binding calculations. We find very strong nonuniform pseudomagnetic fields that can be created by depositing graphene on a substrate decorated with nanoscale pillars and find that the quasiparticle LDOS are strongly modified near the pillars. The optimum configuration of graphene over such nanopillars depends on the imposed boundary conditions. The induced pseudomagnetic fields are larger than $1000 \mathrm{~T}$ and are spatially distributed around the nanopillars. Decreasing the distance between the nanopillars alters the sixfold symmetry of the pseudomagnetic field distribution and results in a new configuration of magnetic confinement for the charge carriers in graphene around the nanopillars. Our study shows the LDOS maps around the pillars, which can be experimentally verified by STM. Here we ignore additional effects possibly arising from electrostatic and/or electron-lattice interactions which could modify the strain configuration or the local density of states.

Atomistic model. A classical atomistic molecular dynamics simulation (MD) is employed to find the optimum configuration of large flakes of graphene (GE) over the nanopillars. The second generation of Brenner's bond-order potential is employed and is able to describe covalent $s p^{3}$ bond breaking and the formation of associated changes in atomic hybridization within a classical potential. ${ }^{13}$ The van der Waals (vdW) interaction between GE and the nanopillars and substrate is modeled by employing the Lennard-Jones (LJ) potential. ${ }^{14-17}$

In order to model the substrate, a (100) surface with a lattice parameter equal to $\ell=3 \AA$ is assumed with LJ parameters $\sigma_{S}$ and $\epsilon_{S}$. The density of sites in the substrate is $\Sigma_{S}=\ell^{-2}$ and the number of atoms is 13700 . As an example we took the nanopillars to be double-wall armchair carbon nanotubes (DWCNTs) taken with $(3,3)$ and $(6,6)$ indexes including 144 atoms (see the left insets in Fig. 1). The number of atoms in the graphene sheet is 44800 , which is equivalent to a sheet of size $34.8 \times 34.43 \mathrm{~nm}^{2}$. We assume that both the substrate and nanopillar atoms are rigid during the simulation.

To model the interaction between two different types of atoms, such as a carbon atom (C) and a substrate atom $(S)$, we adjust the $\mathrm{LJ}$ parameters using the equations $\epsilon_{T}=\sqrt{\epsilon_{\mathrm{C}} \epsilon_{S}}$ and $\sigma_{T}=\left(\sigma_{\mathrm{C}}+\sigma_{S}\right) / 2$. The parameters for carbon are $\sigma_{\mathrm{C}}=$ $3.369 \AA$ and $\epsilon_{\mathrm{C}}=2.63 \mathrm{meV}$. For the substrate atoms we took $\sigma_{S}=3.5 \AA$ and $\epsilon_{S}=10.0 \mathrm{meV}$, which are typical for insulators, e.g., $\mathrm{SiO}_{2} \cdot{ }^{14}$ 

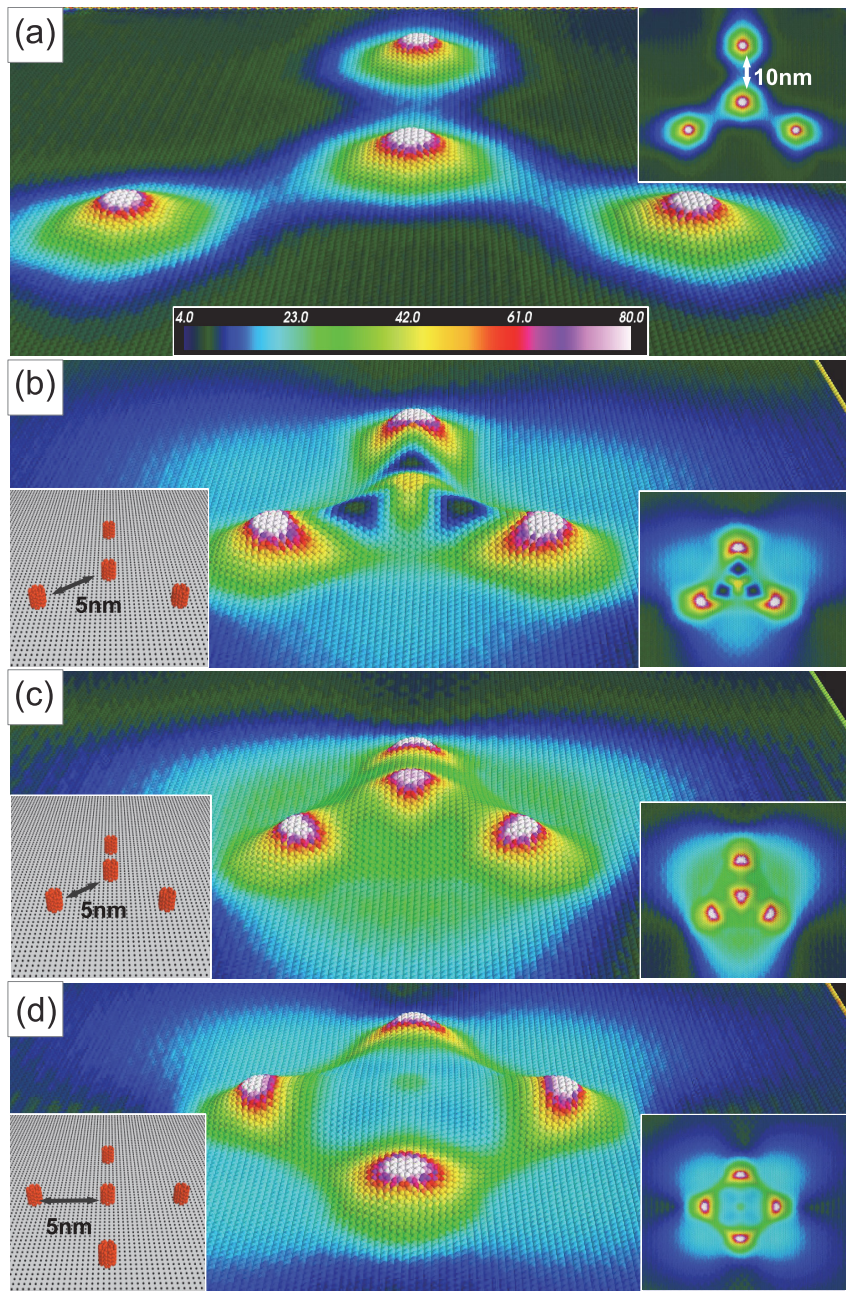

FIG. 1. (Color online) The optimal configuration of graphene on top of double-wall armchair carbon nanotubes (nanopillars) that are place on top of a square lattice substrate (the right insets show a top view). The left insets show the nanopillars and the substrate. In (a) the distance between pillars is $10 \mathrm{~nm}$ while in (c) and (d) it is $5 \mathrm{~nm}$. The pillar heights are $1 \mathrm{~nm}$ in (a), (b), and (d), while the height of the central pillar in (c) is $1.5 \mathrm{~nm}$. The colors indicate the scaled stress distribution, i.e., white represents the highest stress and dark green the lowest stress.

The atomic stress experienced by each $i$ th atom can be expressed as ${ }^{20,21} \eta_{\mu \nu}^{i}=\frac{1}{\Omega}\left(\sum_{j \neq i} r_{i j}^{\nu} F_{i j}^{\mu}\right)$, where the inner summation is over all the carbon atoms which are neighbors of the $i$ th atom which occupies a volume $\Omega=4 \pi a_{0}^{3} / 3$. The scaler $r_{i j}^{v}$ is the $v$ component of the distance between atoms $i$ and $j$ and $F_{i j}^{\mu}$ is the force on $i$ th atom due to atom $j$ th in the $\mu$ direction. We used this expression to calculate the stress on each atom. In order to be able to visualize the stress distribution on the GE atoms, we colored the atoms using a dimensionless invariant quantity ${ }^{22} J_{2}=\frac{1}{6}\left[\left(\eta_{x x}-\eta_{y y}\right)^{2}+\right.$ $\left.\left(\eta_{y y}-\eta_{z z}\right)^{2}+\left(\eta_{z z}-\eta_{x x}\right)^{2}+6\left(\eta_{x y}^{2}+\eta_{x z}^{2}+\eta_{y z}^{2}\right)\right]$, i.e., dark green (white) is related to a minimum (maximum) value of $J_{2}$.

Strain-induced pseudomagnetic field. Coupling the Dirac equation, which governs the low-energy electronics of graphene, to the curved surface is a common way to study the effects of graphene's curved geometries on its corresponding electronic properties. ${ }^{2,8}$ The metric of the curved surface describes the curvature of the surface. The origins of the deformations are external stresses that deform graphene so that the nearest neighbor distances become nonequal. The latter results in modified hopping parameters, which are now a function of the atomic positions $t(\mathbf{r}){ }^{10}$ Assuming small atomic displacements $\left(\mathbf{u}=\mathbf{r}_{i}^{\prime}-\mathbf{r}_{i}<a_{0}\right)$ and rewriting the Dirac Hamiltonian in the effective mass approximation with nonequal hopping parameters yields the induced gauge fields:

$$
\mathbf{A}=\frac{2 \beta \hbar}{3 a_{0} e}\left(u_{x x}-u_{y y},-2 u_{x y}\right),
$$

where $\beta \sim 3$ is a constant and $u_{\alpha \beta}$ is the strain tensor including out-of-plane displacements, ${ }^{2,18}$ i.e.,

$$
u_{\alpha \beta}=\frac{1}{2}\left(\frac{\partial u_{\alpha}}{\partial x_{\beta}}+\frac{\partial u_{\beta}}{\partial x_{\alpha}}+\frac{\partial^{2} h}{\partial x_{\alpha} \partial x_{\beta}}\right),
$$

where $h(x, y)$ is the displacement along the $z$ direction. ${ }^{18}$ Note that the out-of-plane term becomes important when $h$ is comparable to or larger than the $\mathrm{C}-\mathrm{C}$ bond length. ${ }^{19}$ The corresponding pseudomagnetic field component perpendicular to the $x-y$ plane is obtained as

$$
B=\partial_{y} A_{x}-\partial_{x} A_{y} .
$$

This is the pseudomagnetic field that an electron experiences in the $K$ valley. We will find $B$ by making the necessary differentiations numerically in the case of supported boundary conditions. Notice that in Eqs. (1)-(3) the differentiation is discrete and done numerically with the forward differentiation method by using the initially flat and finally strained discreteatomic coordinates. The larger the deformed region, the closer this numerical differentiation is to the continuum limit. ${ }^{9}$

Here we found that the major contribution is due to the out-of-plane terms, which appear mainly around the deformed parts. The other in-plane terms contribute less to the pseudomagnetic field around the deformed parts, particularly when the system is large as compared to the deformed regions.

Tight-binding model. The electronic properties are described by a tight-binding Hamiltonian for the $\pi$ carbon orbitals. The minimal Hamiltonian, which describes the lowenergy band structure is

$$
\mathcal{H}=-\sum_{\langle i, j\rangle, \sigma} t\left(r_{i j}\right) c_{i \sigma}^{\dagger} c_{j \sigma}+\text { H.c. }
$$

where $c_{i \sigma}^{\dagger}\left(c_{j \sigma}\right)$ creates (destroys) an electron at site $i(j)$. The sum runs over nearest neighbors pertaining to opposite sublattices $\langle i, j\rangle$ and the electron spin $\sigma$. In the following we will ignore the spin degrees of freedom since no spin-flipping term is present in the Hamiltonian.

The strain is included in the modified hopping amplitudes between $\pi$ orbitals $t_{\pi}\left(r_{i j}\right)$, according to the empirical relation $t_{\pi}\left(r_{i j}\right)=\gamma_{0} \exp ^{3.37\left(\frac{r_{i j}}{a_{0}}-1\right)}$, where $\gamma_{0}=2.7 \mathrm{eV}$ and $a_{0}=1.42 \AA$ is the equilibrium intercarbon distance..$^{5}$ This also gives a good approximation for the next-nearest neighbor hopping amplitude. We also consider the effect of misalignment of the $\pi$ orbitals due to the finite curvature. This effect translates into the mixing of the $\pi$ and $\sigma$ orbitals. Depending on the local 
curvature, the modified hopping amplitude is

$$
t\left(r_{i j}\right)=t_{\pi} \sin \left(\theta_{i}\right) \sin \left(\theta_{j}\right) \cos (\phi)-t_{\sigma} \cos \left(\theta_{i}\right) \cos \left(\theta_{j}\right),
$$

where $\theta_{i}$ and $\theta_{j}$ are the angles formed by the normals at each atomic position, defined as $\mathbf{n}_{\mathbf{i}}=1 / 3 \sum_{j \neq k}\left(\mathbf{r}_{i j} \times \mathbf{r}_{i k}\right) / \mid \mathbf{r}_{i j} \times$ $\mathbf{r}_{i k} \mid$, with the interatomic distance $\mathbf{r}_{i j}$ and $\phi$ describes the angle formed by the normal $\mathbf{n}_{j}$ and the plane defined by $\mathbf{n}_{i}$ and $\mathbf{r}_{i j}$ (for details, see Refs. 23-25). The strain configuration and the curvature are extracted from the relaxed position of the graphene sheet obtained from our molecular dynamics simulation. For the systems considered here the effect of curvature is small when compared to the effect of strain on the hopping amplitudes.

The system size considered here (44 800 carbon atoms) becomes prohibitively large for an exact diagonalization of the Hamiltonian. Instead we will numerically obtain an approximation of the Green's function by using a Chebyshev expansion within the kernel polynomial method. ${ }^{26-29}$ The Green's function is defined as

$$
G_{i j}(\omega)=\left\langle c_{i}|\hat{G}(\omega)| c_{j}^{\dagger}\right\rangle,
$$

where $\hat{G}(\omega+i \eta)=[\omega+i \eta-\mathcal{H}]^{-1}$.

First a scaling of the excitation energies is performed, e.g., $\tilde{\mathcal{H}}=(\mathcal{H}-\mathbb{1} b) / a, \tilde{\omega}=(\omega-b) / a$, where $a=\left(E_{\max }-\right.$ $\left.E_{\min }\right) /(2-\eta)$ and $b=\left(E_{\max }+E_{\min }\right) / 2$, where $\eta>0$ is a small number. Following Refs. 28 and 26, the components of the Green's function can be expressed as an expansion written in terms of Chebyshev polynomials:

$$
G_{i j}(\tilde{\omega})=\frac{-2 i}{\sqrt{1-\tilde{\omega}^{2}}} \sum_{n=0}^{\infty} a_{n}(i, j) e^{-i n \cdot \arccos (\tilde{\omega})},
$$

where the coefficients $a_{n}(i, j)=\left\langle c_{i} \mid v_{n}\right\rangle$ can be obtained by an iterative procedure involving repeated applications of the Hamiltonian on iterative vectors $\left|v_{n}\right\rangle$ :

$$
\left|v_{n+1}\right\rangle=2 \mathcal{H}\left|v_{n}\right\rangle-\left|v_{n-1}\right\rangle,
$$

where $\left|v_{0}\right\rangle=\left|c_{j}^{\dagger}\right\rangle$ and $\left|v_{-1}\right\rangle=0$. Significant computational speedup is achieved when the computations are done on graphical processing units (GPUs), i.e., video cards. The computations are performed on Nvidia GeForce GTX 580 cards.

The physical properties that can be straightforwardly extracted from the Green's functions are the local density of states (LDOS), $N_{i}(\omega)=-\frac{2}{\pi} \operatorname{Im}\left[G_{i i}(\omega)\right]$, where the factor of 2 appears due to the summation over the spin components.

Results and discussion. At the start of our simulation we put graphene on top of the nanopillars at $h_{0}=1.4 \mathrm{~nm}$. The substrate is at zero height and the nanopillars are located between graphene and the substrate. We have investigated two particular patterns of nanopillars: (i) five DWCNTs which have in-plane coordinates $(0,0)$ and $( \pm d, \pm d)$ with $d=5$ and $10 \mathrm{~nm}$, and (ii) four DWCNTs at $(0,0),( \pm d \sqrt{3} / 2, \pm d / 2)$, and $(0,-d)$. The height of DWCNTs was set to be $1 \mathrm{~nm}$ [except for the central pillar shown in Fig. 1(c) for which the height is $1.5 \mathrm{~nm}$ ]. In order to prevent crumpling at the boundaries we only allow the boundary atoms to vibrate in the $z$ direction.

In Fig. 1 we show the optimal configuration of GE on top of four [Figs. 1(a)-1(c)] and five [Fig. 1(d)] DWCNTs. The right insets in Figs. 1(a)-1(d) show a top view. Notice that the stress distribution is mainly concentrated around the nanopillars, as expected. For the configuration presented in Fig. 1(a) the pillars are $10 \mathrm{~nm}$ apart. Due to the $\mathrm{vdW}$ interaction the graphene sheet will stick to the substrate except around the pillars, where the shape is close to a Gaussian even though deviations from an isotropic description exists. In the atomic limit, a slight anisotropy appears, and the graphene sheet bends mostly in a zigzag direction, making the shape of the deformation hexagonal. In Figs. 1(b)-1(d) the pillars are closer together (i.e., $5 \mathrm{~nm}$ ). Due to its large bending rigidity, the graphene sheet will be suspended over the substrate in the regions between the pillars. Depending on the pillar configuration, various stain configurations are achieved. If all the pillars have the same height [Figs. 1(b) and 1(d)], most of the stress is obtained at the pillar location and where graphene sticks to the substrate. If the central pillar is higher [Fig. 1(c)], besides the maximal stress at the pillar location, high stress is also obtained throughout the suspended sheet.

The corresponding pseudomagnetic field profiles generated by the strain configurations are shown in Figs. 2(a)-2(d). When the deformations are isolated [Fig. 2(a)], the gauge field and the pseudomagnetic field exhibit sixfold symmetry ${ }^{4,10}$ similar to a Gaussian deformation, $h(x, y)=G \exp \left(-\frac{x^{2}+y^{2}}{2 \sigma^{2}}\right)$. The continuum theory predicts that the pseudomagnetic gauge field is $\mathbf{A}=\frac{h(x, y)^{2}}{2 \sigma^{4}}\left(x^{2}-y^{2},-x y\right)$ and the pseudomagnetic field is $B=\frac{h(x, y)^{2}}{\sigma^{6}}\left(x^{2}+y^{2}\right) \sin (3 \theta)$, where $\theta$ is the azimuthal angle. Large pseudomagnetic fields on the order of thousands of teslas are obtained. When the graphene sheet is suspended [Figs. 2(b)-2(d)], the sixfold symmetry survives near the pillars, but more complex pseudomagnetic field profiles can be obtained, from large fields throughout the suspended sheet [Fig. 2(c)] to fields localized only near the edges of the suspended sheet [Fig. 2(d)]. As seen from Figs. 2(c) and 2(d), the closer the pillars, a triangular and rectangular magnetic field profile is created within the position of the pillars. In Figs. 2(c) there is a high magnetic field region at the center and the electron cannot pass through this region.

In order to investigate the effect of the strain on the electronic properties, we input the obtained relaxed positions of the atoms from our atomistic simulations into the tightbinding model in order to find the LDOS maps around the pillars. These are shown in Figs. 2(e)-2(h) for $E=0.1332 \mathrm{eV}$ and in Figs. 2(i)-2(1) for $E=2.2306 \mathrm{eV}$. Two regimes can be observed, depending on the energy. For low energies, the pseudomagnetic field will induce sublattice polarized states localized either near the pillars [Fig. 2(e)] or in the regions with large pseudomagnetic fields [Fig. 2(g)]. In the five-pillar configuration [Fig. 2(h)], these low-energy states are mostly localized near the edges of the suspended region. Interference patterns which depend on the energy are observed. ${ }^{30}$ A very different effect, which is not described by the low-energy Dirac approximation, ${ }^{4}$ is related to the shift of the van Hove singularity seen in unstrained graphene at $E=\gamma_{0}=2.7 \mathrm{eV}$. Because of strain, the hopping parameters will be modified, therefore locally shifting the van Hove singularity. This is observed in Figs. 2(i)-2(1), where the enhancement of the LDOS is correlated with the stress and is enhanced where the stress is larger. Additional interference patterns appear between the pillars. Note the hexagonal symmetry of the 

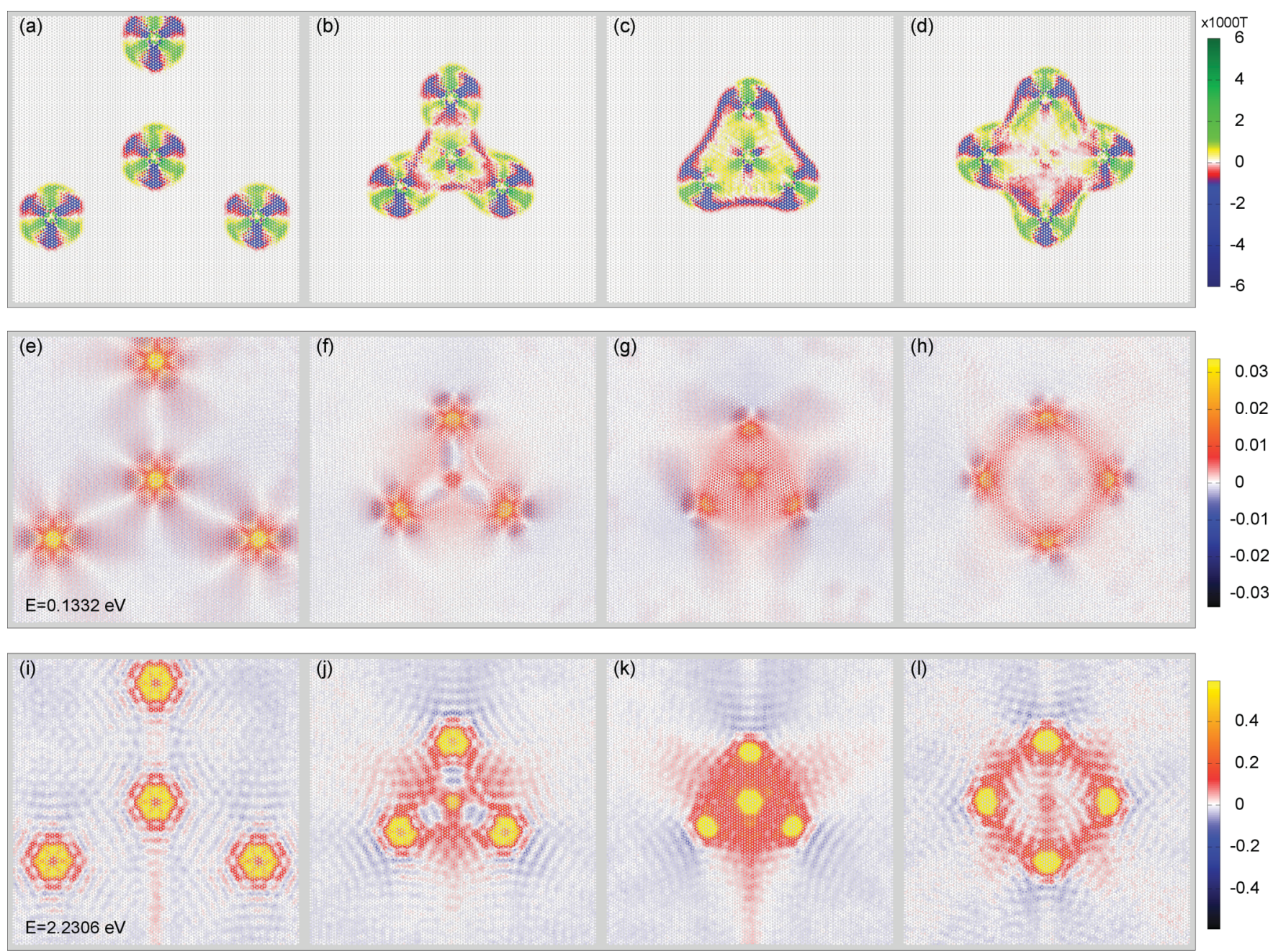

FIG. 2. (Color online) (a)-(d) Pseudomagnetic fields in one Dirac cone for four different pillar configurations shown in Fig. 1, respectively. (e)-(h) Low-energy ( $E=0.1332 \mathrm{eV}$ ) LDOS map and (i)-(l) high-energy ( $E=2.2306 \mathrm{eV}$ ) LDOS map for the same four configurations. Note that the bulk unstrained LDOS is subtracted from the LDOS maps.

LDOS, as seen in Fig. 2(i), showing the deviation from the circular symmetry obtained for an isolated Gaussian bump.

Conclusions. By combining molecular dynamics simulations with tight-binding calculations, we have shown how strain can be manipulated at the nanoscale. Isolated pillars show a sixfold symmetric pseudomagnetic field and LDOS map. By decreasing the distance between the pillars, the sixfold symmetry of the pseudomagnetic field is altered and a complex field profile appears within the suspended area. We found that, by modifying the interpillar distances and the pillar heights, one can design a particular desired magnetic field profile. Modifications of the hopping parameters due to changes in the C-C distances induced by strain modify the LDOS around the deformations of the graphene sheet. Verifications of the sixfold symmetry of the LDOS near pillars could be easily confirmed with STM experiments.

This work was supported by the Flemish Science Foundation (FWO-Vl) and the EuroGRAPHENE project CONGRAN.
${ }^{1}$ K. S. Novoselov, A. K. Geim, S. V. Morozov, D. Jiang, Y. Zhang, S. V. Dubonos, I. V. Grigorieva, and A. A. Firsov, Science 306, 666 (2004).

${ }^{2}$ A. H. Castro Neto, F. Guinea, N. M. R. Peres, K. S. Novoselov, and A. K. Geim, Rev. Mod. Phys. 81, 109 (2009).

${ }^{3}$ N. Levy, S. A. Burke, K. L. Meaker, M. Panlasigui, A. Zettl, F. Guinea, A. H. Castro Neto, and M. F. Crommie, Science 329, 544 (2010).
${ }^{4}$ F. Guinea, M. I. Katsnelson, and A. K. Geim, Nat. Phys. 6, 30 (2010).

${ }^{5}$ V. M. Pereira, A. H. Castro Neto, and N. M. R. Peres, Phys. Rev. B 80, 045401 (2009).

${ }^{6}$ Z. H. Ni, T. Yu, Y. H. Lu, Y. Y. Wang, Y. P. Feng, and Z. X. Shen, ACS Nano 3, 483 (2009).

${ }^{7}$ T. M. G. Mohiuddin, A. Lombardo, R. R. Nair, A. Bonetti, G. Savini, R. Jalil, N. Bonini, D. M. Basko, C. Galiotis, N. Marzari, 
K. S. Novoselov, A. K. Geim, and A. C. Ferrari, Phys. Rev. B 79, 205433 (2009).

${ }^{8}$ F. Guinea, M. I. Katsnelson, and M. A. H. Vozmediano, Phys. Rev. B 77, 075422 (2008).

${ }^{9}$ M. Neek-Amal and F. M. Peeters, Phys. Rev. B 85, 195446 (2012).

${ }^{10}$ K-J. Kim, Ya. M. Blanter, and K.-H. Ahn, Phys. Rev. B 84, 081401(R) (2011).

${ }^{11}$ S. Philip and S. K. Nayak, Appl. Phys. Lett. 94, 032101 (2009).

${ }^{12}$ H. Tomori, A. Kanda, H. Goto, Y. Nukui, Y. Toyota, H. Karube, S. Nihei, Y. Ootuka, K. Tsukagoshi, M. Hayashi, and H. Yoshioka, Appl. Phys. Express 4, 075102 (2011).

${ }^{13}$ D. W. Brenner, O. A. Shenderova, J. A. Harrison, S. J. Stuart, B. Ni, and S. B. Sinnott, J. Phys.: Condens. Matter 14, 783 (2002).

${ }^{14}$ Z.-Y. Ong and E. Pop, Phys. Rev. B 81, 155408 (2010).

${ }^{15}$ M. Neek-Amal, N. Abedpour, S. N. Rasuli, A. Naji, and M. R. Ejtehadi, Phys. Rev. E 82, 051605 (2010).

${ }^{16}$ M. Neek-Amal and F. M. Peeters, Phys. Rev. B 81, 235421 (2010).

${ }^{17}$ A. I. Zhbanov, E. G. Pogorelov, and Y.-C. Chang, ACS Nano 4, 5937 (2010).

${ }^{18}$ M. A. H. Vozmediano, M. I. Katsnelson, and F. Guinea, Phys. Rep. 496, 109 (2010).
${ }^{19}$ L. D. Landau, L. P. Pitaevskii, A. M. Kosevich, and E. M. Lifshitz, Theory of Elasticity (Pergamon, Oxford, UK, 1986).

${ }^{20}$ N. Chandra, S. Namilae, and C. Shet, Phys. Rev. B 69, 094101 (2004).

${ }^{21}$ Q. X. Pei, Y. W. Zhang, and V. B. Shenoy, Carbon 48, 898 (2010).

${ }^{22}$ H. Rafii-Tabar, Phys. Rep. 390, 235 (2004).

${ }^{23}$ J. W. Klos, A. A. Shylau, I. V. Zozoulenko, H. Xu, and T. Heinzel, Phys. Rev. B 80, 245432 (2009).

${ }^{24}$ S. Costamagna, O. Hernandez, and A. Dobry, Phys. Rev. B 81, 115421 (2010).

${ }^{25}$ M. J. Schmidt and D. Loss, Phys. Rev. B 81, 165439 (2010).

${ }^{26}$ A. Weisse, G. Wellein, A. Alvermann, and H. Fehske, Rev. Mod. Phys. 78, 275 (2006).

${ }^{27}$ G. Schubert and H. Fehske, Phys. Rev. Lett. 108, 066402 (2012).

${ }^{28}$ L. Covaci, F. M. Peeters, and M. Berciu, Phys. Rev. Lett. 105, 167006 (2010).

${ }^{29}$ L. Covaci and F. M. Peeters, Phys. Rev. B 84, 241401(R) (2011).

${ }^{30}$ See Supplemental Material at http://link.aps.org/supplemental/ 10.1103/PhysRevB.86.041405 for a movie of the LDOS maps for different energies. 